\title{
Properties of solutions of fourth-order differential equations with boundary conditions
}

\author{
Samir H Saker ${ }^{1}$, Ravi P Agarwal ${ }^{2,3^{*}}$ and Donal O'Regan ${ }^{4}$
}

\section{"Correspondence:}

agarwal@tamuk.edu

${ }^{2}$ Department of Mathematics, Texas

A\&M University-Kingsville, Kingsville,

Texas 78363, USA

${ }^{3}$ Department of Mathematics,

Faculty of Science, King Abdulaziz

University, Jeddah, 21589, Saudi

Arabia

Full list of author information is

available at the end of the article

\begin{abstract}
In this paper, we establish some sufficient conditions for (2,2)-disconjugacy and study the distribution of zeros of nontrivial solutions of fourth-order differential equations. The results are extended to cover some boundary value problems in bending of beams. The main results are proved by making use of a generalization of Hardy's inequality and some Opial-type inequalities. Some examples are considered to illustrate the main results.
\end{abstract}

MSC: $34 \mathrm{~K} 11 ; 34 \mathrm{C} 10$

Keywords: fourth-order differential equations; bending of beams; Opial and Wirtinger inequalities

\section{Introduction}

Linear differential equations subject to some boundary conditions arise in the mathematical description of some physical systems. For example, mathematical models of deflection of beams. These beams, which appear in many structures, deflect under their own weight or under the influence of some external forces. For example, if a load is applied to the beam in a vertical plane containing the axis of symmetry, the beam undergoes a distortion, and the curve connecting the centroids of all cross sections is called the deflection curve or elastic curve. In elasticity it is shown that the deflection of the curve, say $y(x)$ measured from the $x$-axis, approximates the shape of the beam and satisfies the linear fourth-order differential equation

$$
y^{\prime \prime \prime \prime}(x)+q(x) y(x)=0
$$

on an interval, say $[\alpha, \beta]$, with some boundary conditions. Boundary conditions associated with these types of differential equations depend on how the ends of the beams are supported (see [1, 2]). For example, the boundary conditions $y(\alpha)=y^{\prime}(\alpha)=y(\beta)=y^{\prime}(\beta)=0$ correspond to a beam clamped at each end, the boundary conditions $y(\alpha)=y^{\prime}(\alpha)=y^{\prime \prime}(\beta)=$ $y^{\prime \prime \prime}(\beta)=0$ correspond to a beam clamped at $t=\alpha$ and free at $t=\beta$, the boundary conditions $y(\beta)=y^{\prime}(\beta)=y^{\prime \prime}(\alpha)=y^{\prime \prime \prime}(\alpha)=0$ correspond to a beam clamped at $t=\beta$ and free at $t=\alpha$, and the boundary conditions $y(\alpha)=y^{\prime \prime}(\alpha)=y(\beta)=y^{\prime \prime}(\beta)=0$ correspond to a beam hinged or supported at both ends. In this paper, we consider the fourth-order differential

\section{算 Springer}

(c) 2013 Saker et al.; licensee Springer. This is an Open Access article distributed under the terms of the Creative Commons Attribution License (http://creativecommons.org/licenses/by/2.0), which permits unrestricted use, distribution, and reproduction in any medium, provided the original work is properly cited. 
equation

$$
\left(r(t) y^{\prime \prime}(t)\right)^{\prime \prime}-\left(p(t) y^{\prime}(t)\right)^{\prime}+q(t) y(t)=0, \quad t \in I
$$

where $r, p, q: I \rightarrow \mathbb{R}$ are continuous measurable functions such that $r(t)>0$, and $I$ is a nontrivial interval of reals. By a solution of (1.2) on the interval $J \subseteq I$, we mean a nontrivial real-valued function $y \in C^{2}(J)$, which has the property that $r(t) y^{\prime \prime}(t) \in C^{2}(J)$ and satisfies equation (1.2) on $J$. We assume that (1.2) possesses such a nontrivial solution on $I$. Equation (1.2) is said to be $(i, j)$-disconjugate if $i$ and $j$ are positive integers such that $i+j=4$, and no solution of $(1.2)$ has an $(i, j)$-distribution of zeros, i.e., no nontrivial solution has a pair of zeros of multiplicities $i$ and $j$ respectively. If no nontrivial solution of (1.2) has more than three zeros, the equation is termed disconjugate. Equation (1.2) and its generalization were studied in [3-17]. Most of the results in these papers dealt with obtaining sufficient conditions for oscillation, nonoscillation and the types of zeros of solutions, i.e., the solutions that have at least four zeros, two or more of which are distinct zeros and one can determine the disconjugacy type of the equations. To the best of the authors knowledge, there are a few papers [18-20] which studied the distributions (gaps between zeros) of zeros of a special case of (1.2), when $r(t)=1$. Our paper is a continuation of these papers. In particular, we establish some sufficient conditions for $(2,2)$-disconjugacy and establish lower bounds on the distance between zeros of nontrivial solutions and also lower bounds on the distance between zeros of a solution and/or its derivatives. The results in $[18,19]$ extended a result in the literature for disconjugacy of differential equations due to de la Vallée Poussin [21]. This result was established for the linear $n$ th-order differential equation

$$
y^{(n)}(t)+a_{1}(t) y^{(n-1)}(t)+\cdots+a_{n}(t) y(t)=0,
$$

with real continuous coefficients $a_{j}$. In [21] de la Vallée Poussin asserted that equation (1.3) is disconjugate on any interval sufficiently short with respect to the magnitude of the coefficients of the equation. More precisely, he proved that if $\left|a_{j}(t)\right| \leq b_{j}$ on $[\alpha, \beta]$ and the inequality

$$
\sum_{j=1}^{n} \frac{b_{j}(\beta-\alpha)^{j}}{j}<1
$$

holds, then (1.3) is disconjugate.

In [18] the authors studied a special case of (1.2) and considered the equation

$$
y^{(4)}(t)-\left(p(t) y^{\prime}(t)\right)^{\prime}+q(t) y(t)=0, \quad t \in[\alpha, \beta],
$$

and proved that if $y(t)$ is a nontrivial solution of (1.5) which satisfies

$$
y(\alpha)=y^{\prime}(\alpha)=y(\beta)=y^{\prime}(\beta)=0,
$$

then

$$
\frac{\beta-\alpha}{4}\left|\int_{\alpha}^{\beta} p(s) d s\right|+\frac{(\beta-\alpha)^{3}}{192}\left|\int_{\alpha}^{\beta} q(s) d s\right| \geq 1 .
$$


In [19] the authors established some sufficient conditions for (2,2)-disconjugacy of (1.5) and also proved some results on bending of beams. In particular in [19] the authors established: if $y(t)$ is a nontrivial solution of (1.5) which satisfies (1.6), then

$$
\frac{2(\beta-\alpha)^{\frac{1}{2}}}{\pi}\left(\int_{\alpha}^{\beta}\left[P(t)+2 Q_{2}(t)\right]^{2} d t\right)^{\frac{1}{2}}+\left(\int_{\alpha}^{\beta} Q_{1}^{2}(t) d t\right)^{\frac{1}{2}} \frac{(\beta-\alpha)^{\frac{3}{2}}}{\sqrt{48}} \geq 1 .
$$

Our aim in this paper is to employ some new inequalities of Hardy's type and some Opialtype inequalities with weighted functions to establish some new results for the general equation (1.2).

In particular, in this paper, we are concerned with the following problems for the general equation (1.2):

(i) obtain lower bounds for the spacing $\beta-\alpha$, where $y$ is a solution of (1.2) satisfying $y^{(i)}(\alpha)=0$ for $i=0,1,2$ and $y^{\prime \prime}(\beta)=0$,

(ii) obtain lower bounds for the spacing $\beta-\alpha$, where $y$ is a solution of (1.2) satisfying $y^{(i)}(\beta)=0$ for $i=0,1,2$ and $y^{\prime \prime}(\alpha)=0$,

(iii) obtain lower bounds for the spacing $\beta-\alpha$, where $y$ is a solution of (1.2) satisfying $y^{(i)}(\alpha)=0=y^{(i)}(\beta)$ for $i=0,1$.

We also establish some new results related to some boundary value problems in bending of beams. In particular, we consider the boundary conditions (1.6) which correspond to a beam clamped at each end. The case of the boundary conditions

$$
y(\alpha)=y^{\prime}(\alpha)=y^{\prime \prime}(\beta)=r(\beta) y^{\prime \prime \prime}(\beta)-p(\beta) y^{\prime}(\beta)=0
$$

which correspond to a beam clamped at $t=\alpha$ and free at $t=\beta$ and the boundary conditions $y(\beta)=y^{\prime}(\beta)=y^{\prime \prime}(\alpha)=r(\alpha) y^{\prime \prime \prime}(\alpha)-p(\alpha) y^{\prime}(\alpha)=0$ which correspond to a beam clamped at $t=\beta$ and free at $t=\alpha$, and the boundary conditions $y(\alpha)=y^{\prime \prime}(\alpha)=y(\beta)=y^{\prime \prime}(\beta)=0$ which correspond to a beam hinged or supported at both ends will be left to the interested reader.

The rest of the paper is divided into two sections. In Section 2, we prove several results related to the problems (i)-(iii) and also prove some results related to the boundary value problems of the bending of beams with the boundary conditions (1.6) and (1.9). In Section 3, we give some illustrative examples. The results in this paper yield sufficient conditions for disconjugacy.

\section{Main results}

In this section, we prove the main results by using some generalizations of Hardy's inequality and Opial's inequality.

Theorem 2.1 [22, Theorem 3.9.1] Assume that the functions $\vartheta$ and $\phi$ are non-negative and measurable on the interval $(\alpha, \beta), m, n$ are real numbers such that $\mu / m>1$ and $0 \leq$ $k \leq n-1$ but fixed. Let $y(t) \in C^{(n-1)}[\alpha, \beta]$ be such that $y^{(n-1)}(t)$ absolutely continuous on $(\alpha, \beta)$ and $y^{(i)}(\alpha)=0, k \leq i \leq n-1(n \geq 1)$, then

$$
\int_{\alpha}^{\beta} \phi(t)\left|y^{(k)}(t)\right|^{l}\left|y^{(n)}(t)\right|^{m} d t \leq K_{1}(\alpha, \beta)\left[\int_{\alpha}^{\beta} \vartheta(t)\left|y^{(n)}(t)\right|^{\mu} d t\right]^{(l+m) / \mu},
$$


where

$$
\begin{aligned}
& K_{1}(\alpha, \beta):=\frac{\left(\frac{m}{l+m}\right)^{\frac{m}{\mu}}}{((n-k-1) !)^{l}}\left[\int_{\alpha}^{\beta}\left(\frac{\phi^{\mu}(t)}{\vartheta^{m}(t)}\right)^{1 /(\mu-m)}\left(P_{1, k}(t)\right)^{l(\mu-1) /(\mu-m)} d t\right]^{\frac{\mu-m}{\mu}}, \\
& P_{1, k}(t):=\int_{\alpha}^{t}(t-s)^{(n-k-1) \mu /(\mu-1)}(\vartheta(s))^{-1 /(\mu-1)} d s .
\end{aligned}
$$

If we replace $y^{(i)}(\alpha)=0$ by $y^{(i)}(\beta)=0$, then (2.1) holds where $K_{1}$ is replaced by $K_{2}$ which is given by

$$
K_{2}(\alpha, \beta):=\frac{\left(\frac{m}{l+m}\right)^{\frac{m}{\mu}}}{((n-k-1) !)^{l}}\left[\int_{\alpha}^{\beta}\left(\frac{\phi^{\mu}(t)}{\vartheta^{m}(t)}\right)^{1 /(\mu-m)}\left(P_{2, k}(t)\right)^{l(\mu-1) /(\mu-m)} d t\right]^{\frac{\mu-m}{\mu}}
$$

where

$$
P_{2, k}(t):=\int_{t}^{\beta}(s-t)^{(n-k-1) \mu /(\mu-1)}(\vartheta(s))^{-1 /(\mu-1)} d s .
$$

From Theorem 2.1, we note that if $y^{(i)}(\alpha)=y^{(i)}(\beta)=0$, then we have an inequality of the form

$$
\int_{\alpha}^{\beta} \phi(t)\left|y^{(k)}(t)\right|^{l}\left|y^{(n)}(t)\right|^{m} d t \leq K(\alpha, \beta)\left[\int_{\alpha}^{\beta} \vartheta(t)\left|y^{(n)}(t)\right|^{\mu} d t\right]^{(l+m) / \mu},
$$

where in this case $K(\alpha, \beta)$ is determined from the equation

$$
K(\alpha, \beta)=K_{1}(\alpha, \beta)=K_{2}(\alpha, \beta)<\infty .
$$

In the following, we present a Hardy-type inequality [23, 24] that will be used to prove some new results related to the boundary value problems of the bending of beams. If $y$ is absolutely continuous on $(a, b)$ with $y(a)=0$ or $y(b)=0$, then the following inequality holds:

$$
\left(\int_{a}^{b} p(t)|y(t)|^{n} d t\right)^{\frac{1}{n}} \leq C\left(\int_{a}^{b} r(t)\left|y^{\prime}(t)\right|^{m} d t\right)^{\frac{1}{m}},
$$

where $q, r$ the weighted functions are measurable positive functions in the interval $(a, b)$ and $m, n$ are real parameters satisfying $0<n \leq \infty$ and $1 \leq m \leq \infty$. The constant $C$ satisfies

$$
C \leq k(m, n) A \text { for } 1<m \leq n,
$$

where $k(m, n)=n^{1 / m}\left(m^{*}\right)^{1 / m^{*}}$ and $m^{*}=\frac{m}{m-1}$ and

$$
\begin{aligned}
& A:=A(a)=\sup _{a<t<b}\left(\int_{t}^{b} p(t) d t\right)^{\frac{1}{n}}\left(\int_{a}^{t} r^{1-m^{*}}(s) d s\right)^{1 / m^{*}} \quad \text { if } y(a)=0, \\
& A:=A(b)=\sup _{a<t<b}\left(\int_{a}^{t} p(t) d t\right)^{\frac{1}{n}}\left(\int_{t}^{b} r^{1-m^{*}}(s) d s\right)^{1 / m^{*}} \quad \text { if } y(b)=0 .
\end{aligned}
$$


An inequality of type (2.6) also holds when $y(a)=y(b)=0$. In this case, we see that (2.6) is satisfied if $C \leq k(m, n) A(a, b)$, where

$$
\begin{aligned}
A(a, b)= & \sup _{(c, d) \subset(a, b)}\left(\int_{c}^{d} p(t) d t\right)^{\frac{1}{n}} \\
& \times \min \left\{\left(\int_{a}^{c} r^{1-m^{*}}(s) d s\right)^{1 / m^{*}},\left(\int_{d}^{b} r^{1-m^{*}}(s) d s\right)^{1 / m^{*}}\right\} .
\end{aligned}
$$

For simplicity, we let

$$
\begin{aligned}
A(r)= & \sup _{(c, d) \subset(\alpha, \beta)}\left(\int_{c}^{d} r(t) d t\right)^{\frac{1}{2}} \\
& \times \min \left\{\left(\int_{\alpha}^{c} \frac{1}{r(s)} d s\right)^{1 / 2},\left(\int_{d}^{\beta} \frac{1}{r(s)} d s\right)^{1 / 2}\right\} .
\end{aligned}
$$

Now, we are ready to state and prove the main results. For simplicity, we introduce the following notations:

$$
\left.\begin{array}{lc}
\Phi_{1}(Q, r):=\frac{1}{2 \sqrt{2}}\left[\int_{\alpha}^{\beta} \frac{Q^{2}(t)}{r(t)} P_{1,0}(t) d t\right]^{\frac{1}{2}}, & P_{1,0}(t):=\int_{\alpha}^{t} \frac{(t-s)^{4}}{r(s)} d s, \\
\Psi_{1}(Q, r):=\sqrt{\frac{1}{2}}\left[\int_{\alpha}^{\beta} \frac{Q^{2}(t)}{r(t)} P_{1,1}(t)\right]^{\frac{1}{2}}, & P_{1,1}(t)=\int_{\alpha}^{t} \frac{1}{r(s)} d s, \\
\Pi_{1}(p, r):=\frac{1}{\sqrt{2}}\left[\int_{\alpha}^{\beta} \frac{p^{2}(t)}{r(t)} P_{1}(t) d t\right]^{\frac{1}{2}}, & P_{1}(t):=\int_{\alpha}^{t} \frac{(t-s)^{2}}{r(s)} d s, \\
\Lambda_{1}\left(r, r^{\prime}\right):=\sqrt{\frac{1}{2}}\left[\int_{\alpha}^{\beta} \frac{\left(r^{\prime}(t)\right)^{2}}{r(t)} P_{1,2}(t) d t\right]^{\frac{1}{2}}, & P_{1,2}(t)=\int_{\alpha}^{t} \frac{1}{r(s)} d s,
\end{array}\right\}
$$

and

$$
\left.\begin{array}{lc}
\Phi_{2}(Q, r):=\frac{1}{2 \sqrt{2}}\left[\int_{\alpha}^{\beta} \frac{Q^{2}(t)}{r(t)} P_{2,0}(t) d t\right]^{\frac{1}{2}}, & P_{2,0}(t):=\int_{t}^{\beta} \frac{(s-t)^{4}}{r(s)} d s, \\
\Psi_{2}(Q, r):=\sqrt{\frac{1}{2}}\left[\int_{\alpha}^{\beta} \frac{Q^{2}(t)}{r(t)} P_{2,1}(t)\right]^{\frac{1}{2}}, & P_{2,1}(t)=\int_{t}^{\beta} \frac{1}{r(s)} d s, \\
\Pi_{2}(p, r):=\frac{1}{\sqrt{2}}\left[\int_{\alpha}^{\beta} \frac{p^{2}(t)}{r(t)} P_{2}(t) d t\right]^{\frac{1}{2}}, & P_{2}(t):=\int_{t}^{\beta} \frac{(s-t)^{2}}{r(s)} d s, \\
\Lambda_{2}\left(r, r^{\prime}\right):=\sqrt{\frac{1}{2}}\left[\int_{\alpha}^{\beta} \frac{\left(r^{\prime}(t)\right)^{2}}{r(t)} P_{2,2}(t) d t\right]^{\frac{1}{2}}, & P_{2,2}(t):=\int_{t}^{\beta} \frac{1}{r(s)} d s .
\end{array}\right\}
$$

Theorem 2.2 Suppose that $y$ is a nontrivial solution of $(1.2)$. If $y^{(i)}(\alpha)=0$ for $i=0,1,2$ and $y^{\prime \prime}(\beta)=0$, then

$$
\Phi_{1}(Q, r)+\Psi_{1}^{*}(Q, r)+\Pi_{1}(p, r)+\Lambda_{1}\left(P, r, P_{1,2}\right) \geq 1
$$

where $Q(t)=\int_{t}^{\beta} q(s) d s$ and $\Psi_{1}^{*}(Q, r)=4 A^{2}(r) \Psi_{1}(Q, r)$. If $y^{(i)}(\beta)=0$ for $i=0,1,2$ and $y^{\prime \prime}(\alpha)=$ 0 , then

$$
\Phi_{2}(Q, r)+\Psi_{2}^{*}(Q, r)+\Pi_{2}(p, r)+\Lambda_{2}\left(P, r, P_{2,2}\right) \geq 1,
$$

where $Q(t)=\int_{\alpha}^{t} q(s) d s$ and $\Psi_{2}^{*}(Q, r)=4 A^{2}(r) \Psi_{2}(Q, r)$. 
Proof We prove (2.12). Multiplying (1.2) by $y^{\prime \prime}(t)$ and integrating by parts yield

$$
\begin{aligned}
\int_{\alpha}^{\beta}\left(r(t) y^{\prime \prime}(t)\right)^{\prime \prime} y^{\prime \prime}(t) d t & =\left.\left(r(t) y^{\prime \prime}(t)\right)^{\prime} y^{\prime \prime}(t)\right|_{\alpha} ^{\beta}-\int_{\alpha}^{\beta}\left(r(t) y^{\prime \prime}(t)\right)^{\prime} y^{\prime \prime \prime}(t) d t \\
& =\int_{\alpha}^{\beta}\left(p(t) y^{\prime}(t)\right)^{\prime} y^{\prime \prime}(t) d t-\int_{\alpha}^{\beta} q(t) y(t) y^{\prime \prime}(t) d t .
\end{aligned}
$$

This with $y^{\prime \prime}(\alpha)=y^{\prime \prime}(\beta)=0$ and $Q(t)=\int_{t}^{\beta} q(s) d s$ yields

$$
\begin{aligned}
\int_{\alpha}^{\beta} r(t)\left(y^{\prime \prime \prime}(t)\right)^{2} d t= & -\int_{\alpha}^{\beta} r^{\prime}(t) y^{\prime \prime}(t) y^{\prime \prime \prime}(t) d t-\int_{\alpha}^{\beta}\left(p(t) y^{\prime}(t)\right)^{\prime} y^{\prime \prime}(t) d t \\
& -\int_{\alpha}^{\beta} Q^{\prime}(t) y(t) y^{\prime \prime}(t) d t .
\end{aligned}
$$

Integrating by parts the term $\int_{\alpha}^{\beta}\left(p(t) y^{\prime}(t)\right)^{\prime} y^{\prime \prime}(t) d t$, we see that

$$
\int_{\alpha}^{\beta}\left(p(t) y^{\prime}(t)\right)^{\prime} y^{\prime \prime}(t) d t=\left.p(t) y^{\prime}(t) y^{\prime \prime}(t)\right|_{\alpha} ^{\beta}-\int_{\alpha}^{\beta} p(t) y^{\prime}(t) y^{\prime \prime \prime}(t) d t .
$$

Using the assumption $y^{\prime \prime}(\alpha)=y^{\prime \prime}(\beta)=0$, we have

$$
-\int_{\alpha}^{\beta}\left(p(t) y^{\prime}(t)\right)^{\prime} y^{\prime \prime}(t) d t=\int_{\alpha}^{\beta} p(t) y^{\prime}(t) y^{\prime \prime \prime}(t) d t
$$

Integrating by parts the term $\int_{\alpha}^{\beta} Q^{\prime}(t) y^{\prime \prime}(t) y(t) d t$ yields

$$
\begin{aligned}
\int_{\alpha}^{\beta} Q^{\prime}(t) y^{\prime \prime}(t) y(t) d t= & \left.Q(t) y^{\prime \prime}(t) y(t)\right|_{\alpha} ^{\beta}-\int_{\alpha}^{\beta} Q(t) y^{\prime}(t) y^{\prime \prime}(t) d t \\
& -\int_{\alpha}^{\beta} Q(t) y(t) y^{\prime \prime \prime}(t) d t .
\end{aligned}
$$

Using the assumption $y^{\prime \prime}(\beta)=y^{\prime \prime}(\alpha)=0$, we obtain

$$
-\int_{\alpha}^{\beta} Q^{\prime}(t) y^{\prime \prime}(t) y(t) d t=\int_{\alpha}^{\beta} Q(t) y^{\prime}(t) y^{\prime \prime}(t) d t+\int_{\alpha}^{\beta} Q(t) y(t) y^{\prime \prime \prime}(t) d t .
$$

Substituting (2.17) and (2.16) into (2.15) yields

$$
\begin{aligned}
\int_{\alpha}^{\beta} r(t)\left|y^{\prime \prime \prime}(t)\right|^{2} d t \leq & \int_{\alpha}^{\beta}|p(t)|\left|y^{\prime}(t)\right|\left|y^{\prime \prime \prime}(t)\right| d t+\int_{\alpha}^{\beta}|Q(t)|\left|y^{\prime}(t)\right|\left|y^{\prime \prime}(t)\right| d t \\
& +\int_{\alpha}^{\beta}|Q(t)||y(t)|\left|y^{\prime \prime \prime}(t)\right| d t+\int_{\alpha}^{\beta}\left|r^{\prime}(t)\right|\left|y^{\prime \prime}(t)\right|\left|y^{\prime \prime \prime}(t)\right| d t .
\end{aligned}
$$

Applying the inequality (2.1) on the integral $\int_{\alpha}^{\beta}|Q(t)||y(t)|\left|y^{\prime \prime \prime}(t)\right| d t$, with $\phi(t)=|Q(t)|$, $\vartheta(t)=r(t), m=1, k=0, l=1, n=3$ and $\mu=2$, we get (note that $y^{(i)}(\alpha)=0$ for $i=0,1,2$ ) that

$$
\int_{\alpha}^{\beta}|Q(t)| y(t)\left|y^{\prime \prime \prime}(t)\right| d t \leq \Phi_{1}(Q, r) \int_{\alpha}^{\beta} r(t)\left|y^{\prime \prime \prime}(t)\right|^{2} d t
$$


where $\Phi_{1}(Q, r)$ is defined as in (2.10). Applying the inequality (2.1) again on the integral

$$
\int_{\alpha}^{\beta}|Q(t)|\left|y^{\prime}(t)\right|\left|y^{\prime \prime}(t)\right| d t
$$

with $\phi(t)=Q(t), \vartheta(t)=r(t), k=1, n=2, l=m=1$ and $\mu=2$, we see that

$$
\int_{\alpha}^{\beta}|Q(t)|\left|y^{\prime}(t)\right|\left|y^{\prime \prime}(t)\right| d t \leq \Psi_{1}(Q, r) \int_{\alpha}^{\beta} r(t)\left|y^{\prime \prime}(t)\right|^{2} d t,
$$

where $\Psi_{1}(Q, r)$ is defined as in (2.10). Applying the inequality (2.6) on the integral $\int_{\alpha}^{\beta} r(t)\left|y^{\prime \prime}(t)\right|^{2} d t$ (note $\left.y^{\prime \prime}(\alpha)=0=y^{\prime \prime}(\beta)\right)$, we see that

$$
\int_{\alpha}^{\beta} r(t)\left|y^{\prime \prime}(t)\right|^{2} d t \leq 4 A^{2}(r) \int_{\alpha}^{\beta} r(t)\left|y^{\prime \prime \prime}(t)\right|^{2} d t
$$

Substituting (2.21) into (2.20), we have

$$
\int_{\alpha}^{\beta}|Q(t)|\left|y^{\prime}(t)\right|\left|y^{\prime \prime}(t)\right| d t \leq \Psi_{1}^{*}(Q, r) \int_{\alpha}^{\beta} r(t)\left|y^{\prime \prime \prime}(t)\right|^{2} d t
$$

Applying the inequality (2.1) on the integral $\int_{\alpha}^{\beta}|p(t)|\left|y^{\prime}(t)\right|\left|y^{\prime \prime \prime}(t)\right| d t$, with $\phi(t)=|p(t)|$, $\vartheta(t)=r(t), m=1, k=1, l=1, n=3$ and $\mu=2$, we get (note that $y^{(i)}(\alpha)=0$ for $i=0,1,2$ ) that

$$
\int_{\alpha}^{\beta}|p(t)| y^{\prime}(t)\left|y^{\prime \prime \prime}(t)\right| d t \leq \Pi_{1}(p, r) \int_{\alpha}^{\beta} r(t)\left|y^{\prime \prime \prime}(t)\right|^{2} d t,
$$

where $\Pi_{1}(p, r)$ is defined as in (2.10). Applying the inequality (2.1) again on the integral

$$
\int_{\alpha}^{\beta}\left|p^{\prime}(t)\right|\left|y^{\prime \prime}(t)\right|\left|y^{\prime \prime \prime}(t)\right| d t
$$

with $\phi(t)=p^{\prime}(t), \vartheta(t)=r(t), k=2, n=3, l=m=1$ and $\mu=2$, we see that

$$
\int_{\alpha}^{\beta}\left|p^{\prime}(t)\right|\left|y^{\prime}(t)\right|\left|y^{\prime \prime}(t)\right| d t \leq \Lambda_{1}\left(r, r^{\prime}\right)\left[\int_{\alpha}^{\beta} r(t)\left|y^{\prime \prime \prime}(t)\right|^{2} d t\right],
$$

where $\Lambda_{1}\left(r, r^{\prime}\right)$ is defined as in (2.10). Substituting (2.19), (2.22), (2.23) and (2.24) into (2.18) and canceling the term $\int_{\alpha}^{\beta} r(t)\left|y^{\prime \prime \prime}(t)\right|^{2} d t$, we have

$$
\Phi_{1}(Q, r,)+\Psi_{1}^{*}(Q, r)+\Pi_{1}(p, r)+\Lambda_{1}\left(r, r^{\prime}\right) \geq 1,
$$

which is the desired inequality (2.12). The proof of (2.13) is similar to the proof of (2.12) by using integration by parts, $K_{1}(\alpha, \beta)$ is replaced by $K_{2}(\alpha, \beta)$ and $\Phi_{2}(Q, r), \Psi_{2}^{*}(Q, r), \Pi_{2}(p, r)$ and $\Lambda_{2}\left(r, r^{\prime}\right)$ which are defined in (2.11) instead of the constants $\Phi_{1}(Q, r), \Psi_{1}^{*}(Q, r), \Pi_{1}(p, r)$ and $\Lambda_{1}\left(r, r^{\prime}\right)$. The proof is complete.

In the following, we apply a special case of an inequality with two functions proved by Agarwal and Pang [25]. In this case $\Psi_{1}^{*}(Q, r)$ and $\Psi_{2}^{*}(Q, r)$ will be replaced by $C_{2}$ and $C_{2}^{*}$ defined below. This inequality is given in the following theorem. 
Theorem 2.3 [25] Let $c(t), r(t)$ be non-negative measurable functions on $(\alpha, \beta)$ and $0 \leq$ $k \leq n-1(n \geq 1)$ but fixed. If $y(t) \in C^{n-1}[\alpha, \beta]$ such that $y^{(i)}(\alpha)=0, k \leq i \leq n-1, y^{(n-1)}$ is absolutely continuous on $(\alpha, \beta)$, then

$$
\int_{\alpha}^{\beta} c(t)\left|y^{(k)}(t)\right|\left|y^{(k+1)}(t)\right| d t \leq C_{\alpha} \int_{\alpha}^{\beta} r(t)\left|y^{(n)}(t)\right|^{2} d t,
$$

where

$$
C_{\alpha}:=\frac{1}{2((n-k-1) !)^{2}} \max _{t \in[\alpha, \beta]} c(t) \int_{\alpha}^{\beta} \frac{(s-\alpha)^{2(n-k-1)}}{r(s)} d s .
$$

If $y(t) \in C^{n-1}[\alpha, \beta]$ such that $y^{(i)}(\beta)=0, k \leq i \leq n-1, y^{(n-1)}$ is absolutely continuous on $(\alpha, \beta)$, then (2.25) holds with $C_{\alpha}$ is replaced by $C_{\beta}$ where

$$
C_{\beta}:=\frac{1}{2((n-k-1) !)^{2}} \max _{t \in[\alpha, \beta]} c(t) \int_{\alpha}^{\beta} \frac{(\beta-s)^{2(n-k-1)}}{r(s)} d s
$$

Now we apply the inequality (2.25). Suppose that the solution $y(t)$ of $(1.2)$ satisfies $y^{\prime}(\alpha)=$ $y^{\prime \prime}(\alpha)=0$. Applying the inequality (2.25) with $c(t)=Q(t), k=1$ and $n=3$ on the term $\int_{\alpha}^{\beta}|Q(t)|\left|y^{\prime}(t)\right|\left|y^{\prime \prime}(t)\right| d t$, we obtain

$$
\int_{\alpha}^{\beta}|Q(t)|\left|y^{\prime}(t)\right|\left|y^{\prime \prime}(t)\right| d t \leq C_{2} \int_{\alpha}^{\beta} r(t)\left|y^{\prime \prime \prime}(t)\right|^{2} d t
$$

where

$$
C_{2}:=\frac{1}{2} \max _{t \in[\alpha, \beta]}|Q(t)| \int_{\alpha}^{\beta} \frac{(s-\alpha)^{2}}{r(s)} d s
$$

If instead $y^{\prime}(\beta)=y^{\prime \prime}(\beta)=0$, then (2.26) holds where $C_{2}$ is replaced by

$$
C_{2}^{*}:=\frac{1}{2} \max _{t \in[\alpha, \beta]}|Q(t)| \int_{\alpha}^{\beta} \frac{(\beta-s)^{2}}{r(s)} d s .
$$

Using $C_{2}$ and $C_{2}^{*}$ instead of $\Psi_{1}(Q, r)$ and $\Psi_{2}(Q, r)$ in the proof of Theorem 2.2, we obtain the following result.

Theorem 2.4 Suppose that $y$ is a nontrivial solution of $(1.2)$. If $y^{(i)}(\alpha)=0$ for $i=0,1,2$ and $y^{\prime \prime}(\beta)=0$, then

$$
\Phi_{1}(Q, r)+\Pi_{1}(p, r)+\frac{1}{2} \max _{t \in[\alpha, \beta]}|Q(t)| \int_{\alpha}^{\beta} \frac{(s-\alpha)^{2}}{r(s)} d s+\Lambda_{1}\left(r, r^{\prime}\right) \geq 1
$$

where $Q(t)=\int_{t}^{\beta} q(s) d s$. If $y^{(i)}(\beta)=0$ for $i=0,1,2$ and $y^{\prime \prime}(\alpha)=0$, then

$$
\Phi_{2}(Q, r)+\Pi_{2}(p, r)+\frac{1}{2} \max _{t \in[\alpha, \beta]}|Q(t)| \int_{\alpha}^{\beta} \frac{(\beta-s)^{2}}{r(s)} d s+\Lambda_{2}\left(r, r^{\prime}\right) \geq 1
$$

where $Q(t)=\int_{\alpha}^{t} q(s) d s$. 
If the function $r(t)$ is non-increasing on $[\alpha, \beta]$, then

$$
\left[\int_{\alpha}^{\beta} \frac{(s-\alpha)^{2}}{r(s)} d s\right] \leq \frac{1}{r(\beta)}\left[\int_{\alpha}^{\beta}(s-\alpha)^{2} d s\right]=\frac{(\beta-\alpha)^{3}}{3 r(\beta)},
$$

and

$$
\left[\int_{\alpha}^{\beta} \frac{(\beta-s)^{2}}{r(s)} d s\right] \leq \frac{1}{r(\beta)}\left[\int_{\alpha}^{\beta}(\beta-s)^{2} d s\right]=\frac{(\beta-\alpha)^{3}}{3 r(\beta)} .
$$

Substituting these last two inequalities into Theorem 2.4, we have the following result.

Theorem 2.5 Assume that $r(t)$ is a non-increasing function. If y is a nontrivial solution of (1.2) which satisfies $y^{(i)}(\alpha)=0$ for $i=0,1,2$ and $y^{\prime \prime}(\beta)=0$, then

$$
\Phi_{1}(Q, r)+\Pi_{1}(p, r)+\frac{(\beta-\alpha)^{3}}{6 r(\beta)} \max _{t \in[\alpha, \beta]}\left|\int_{t}^{\beta} q(s) d s\right|+\Lambda_{1}\left(r, r^{\prime}\right) \geq 1 .
$$

If instead $y^{(i)}(\beta)=0$ for $i=0,1,2$ and $y^{\prime \prime}(\alpha)=0$, then

$$
\Phi_{2}(Q, r)+\Pi_{2}(p, r)+\frac{(\beta-\alpha)^{3}}{6 r(\beta)} \max _{t \in[\alpha, \beta]}\left|\int_{\alpha}^{t} q(s) d s\right|+\Lambda_{2}\left(r, r^{\prime}\right) \geq 1 .
$$

In the following, we apply an inequality due to Boyd [26] to obtain new results. The Boyd inequality states that if $y \in C^{1}[a, b]$ with $y(a)=0$ (or $\left.y(b)=0\right)$, then

$$
\int_{a}^{b}|y(t)|^{v}\left|y^{\prime}(t)\right|^{\eta} d t \leq N(v, \eta, s)(b-a)^{v}\left(\int_{a}^{b}\left|y^{\prime}(t)\right|^{s} d t\right)^{\frac{v+\eta}{s}},
$$

where $v>0, s>1,0 \leq \eta<s$,

$$
N(v, \eta, s):=\frac{(s-\eta) v^{v} \sigma^{v+\eta-s}}{(s-1)(v+\eta)(I(\nu, \eta, s))^{v}}, \quad \sigma:=\left\{\frac{v(s-1)+(s-\eta)}{(s-1)(v+\eta)}\right\}^{\frac{1}{s}}
$$

and

$$
I(\nu, \eta, s):=\int_{0}^{1}\left\{1+\frac{s(\eta-1)}{s-\eta} t\right\}^{-(v+\eta+s v) / s v}[1+(\eta-1) t] t^{1 / v-1} d t
$$

Note that an inequality of type (2.27) also holds when $y(a)=y(b)=0$. Choose $c=(a+b) / 2$ and apply (2.27) to $[a, c]$ and $[c, b]$ and then add to obtain

$$
\int_{a}^{b}|y(t)|^{v}\left|y^{\prime}(t)\right|^{\eta} d t \leq N(v, \eta, s)\left(\frac{b-a}{2}\right)^{v}\left(\int_{a}^{b}\left|y^{\prime}(t)\right|^{s} d t\right)^{\frac{v+\eta}{s}},
$$

where $N(v, \eta, s)$ is defined as in (2.28). An inequality of type (2.27) holds when $\eta=s$ when $y(a)=0$ (or $y(b)=0)$. In this case, equation (2.27) becomes

$$
\int_{a}^{b}|y(t)|^{v}\left|y^{\prime}(t)\right|^{\eta} d t \leq L(v, \eta)(b-a)^{v}\left(\int_{a}^{b}\left|y^{\prime}(t)\right|^{\eta} d t\right)^{\frac{v+\eta}{\eta}},
$$


Saker et al. Journal of Inequalities and Applications 2013, 2013:278

Page 10 of 15

where

$$
L(v, \eta):=\frac{\eta v^{\eta}}{v+\eta}\left(\frac{v}{v+\eta}\right)^{\frac{v}{\eta}}\left(\frac{\Gamma\left(\frac{\eta+1}{\eta}+\frac{1}{v}\right)}{\Gamma\left(\frac{\eta+1}{\eta}\right) \Gamma\left(\frac{1}{v}\right)}\right)^{v}
$$

and $\Gamma$ is the gamma function. To apply (2.30) on the term

$$
\int_{\alpha}^{\beta}|Q(t)|\left|y^{\prime}(t)\right|\left|y^{\prime \prime}(t)\right| d t
$$

we first apply the Schwarz inequality

$$
\int_{\alpha}^{\beta}|f(t) g(t)| d t \leq\left(\int_{\alpha}^{\beta}|f(t)|^{2} d t\right)^{\frac{1}{2}} \times\left(\int_{\alpha}^{\beta}|g(t)|^{2} d t\right)^{\frac{1}{2}}
$$

to get that

$$
\int_{\alpha}^{\beta}|Q(t)|\left|y^{\prime}(t)\right|\left|y^{\prime \prime}(t)\right| d t \leq\left(\int_{\alpha}^{\beta}|Q(t)|^{2} d t\right)^{\frac{1}{2}}\left(\int_{\alpha}^{\beta}\left|y^{\prime}(t)\right|^{2}\left|y^{\prime \prime}(t)\right|^{2} d t\right)^{\frac{1}{2}} .
$$

Now, we apply the inequality (2.30) on the integral $\int_{\alpha}^{\beta}\left|y^{\prime}(t)\right|^{2}\left|y^{\prime \prime}(t)\right|^{2} d t$ with $v=\eta=2$ (note that $\left.y^{\prime}(\alpha)=0\right)$. This gives us that

$$
\int_{\alpha}^{\beta}\left|y^{\prime}(t)\right|^{2}\left|y^{\prime \prime}(t)\right|^{2} d t \leq \frac{4(\beta-\alpha)^{2}}{\pi^{2} r^{2}(\beta)}\left[\int_{\alpha}^{\beta} r(t)\left|y^{\prime \prime}(t)\right|^{2} d t\right]^{2}
$$

where we assumed that $r(t)$ is a non-increasing function. Substituting (2.34) into (2.33), we have

$$
\begin{aligned}
& \int_{\alpha}^{\beta}|Q(t)|\left|y^{\prime}(t)\right|\left|y^{\prime \prime}(t)\right| d t \\
& \quad \leq \frac{2}{\pi} \frac{(\beta-\alpha)}{r(\beta)}\left(\int_{\alpha}^{\beta}|Q(t)|^{2} d t\right)^{\frac{1}{2}} \int_{\alpha}^{\beta} r(t)\left|y^{\prime \prime}(t)\right|^{2} d t .
\end{aligned}
$$

Applying the inequality (2.6) on the integral $\int_{\alpha}^{\beta} r(t)\left|y^{\prime \prime}(t)\right|^{2} d t$, where $y^{\prime \prime}(\alpha)=0=y^{\prime \prime}(\beta)$, we see that

$$
\int_{\alpha}^{\beta} r(t)\left|y^{\prime \prime}(t)\right|^{2} d t \leq A^{2}(r) \int_{\alpha}^{\beta} r(t)\left|y^{\prime \prime \prime}(t)\right|^{2} d t
$$

This implies that

$$
\begin{aligned}
& \int_{\alpha}^{\beta}|Q(t)|\left|y^{\prime}(t)\right|\left|y^{\prime \prime}(t)\right| d t \\
& \quad \leq \frac{2}{\pi} \frac{(\beta-\alpha)}{r(\beta)} A^{2}(r)\left(\int_{\alpha}^{\beta}|Q(t)|^{2} d t\right)^{\frac{1}{2}} \int_{\alpha}^{\beta} r(t)\left|y^{\prime \prime \prime}(t)\right|^{2} d t,
\end{aligned}
$$


where $Q(t)=\int_{t}^{\beta} q(s) d s$. If we replace $y^{\prime}(\alpha)=0$ by $y^{\prime}(\beta)=0$, then (2.35) becomes

$$
\begin{aligned}
& \int_{\alpha}^{\beta}|Q(t)|\left|y^{\prime}(t)\right|\left|y^{\prime \prime}(t)\right| d t \\
& \quad \leq \frac{2}{\pi} \frac{(\beta-\alpha)}{r(\beta)} A^{2}(r)\left(\int_{\alpha}^{\beta}|Q(t)|^{2} d t\right)^{\frac{1}{2}} \int_{\alpha}^{\beta} r(t)\left|y^{\prime \prime \prime}(t)\right|^{2} d t,
\end{aligned}
$$

where $Q(t)=\int_{\alpha}^{t} q(s) d s$.

Using the inequalities (2.34) and (2.36) and proceeding as in the proof of Theorem 2.4, we obtain the following result.

Theorem 2.6 Assume that $r(t)$ is a non-increasing function. If y is a nontrivial solution of (1.2) such that $y^{(i)}(\alpha)=0$ for $i=0,1,2$ and $y^{\prime \prime}(\beta)=0$, then

$$
\Phi_{1}(Q, r)+\Pi_{1}(p, r)+\Lambda_{1}\left(r, r^{\prime}\right)+\frac{2}{\pi} \frac{(\beta-\alpha)}{r(\beta)} A^{2}(r)\left(\int_{\alpha}^{\beta}|Q(t)|^{2} d t\right)^{\frac{1}{2}} \geq 1,
$$

where $Q(t)=\int_{t}^{\beta} q(s) d s$. If instead $y^{(i)}(\beta)=0$ for $i=0,1,2$ and $y^{\prime \prime}(\alpha)=0$, then

$$
\Phi_{2}(Q, r)+\Pi_{2}(p, r)+\Lambda_{2}\left(r, r^{\prime}\right)+\frac{2}{\pi} \frac{(\beta-\alpha)}{r(\beta)} A^{2}(r)\left(\int_{\alpha}^{\beta}|Q(t)|^{2} d t\right)^{\frac{1}{2}} \geq 1,
$$

where $Q(t)=\int_{\alpha}^{t} q(s) d s$.

Remark 1 In Theorem 2.6, if $y^{\prime}(\alpha)=y^{\prime}(\beta)$, then the term $\frac{2}{\pi} \frac{(\beta-\alpha)}{r(\beta)}$ changes to $\sqrt{\frac{1}{\pi}} \frac{(\beta-\alpha)}{r(\beta)}$. We note also that the inequalities in Theorem 2.6 hold for any antiderivative $Q$. Note

$$
Q^{*}=\inf _{k} \int_{\alpha}^{\beta}(Q(t)+k)^{2} d t=\int_{\alpha}^{\beta} Q^{2}(t) d t-\frac{1}{\beta-\alpha}\left(\int_{\alpha}^{\beta} Q(t) d t\right)^{2},
$$

the minimum being attained at $k=\frac{-1}{\beta-\alpha} \int_{\alpha}^{\beta} Q(t) d t$. Now, one can use this in Theorem 2.6 to obtain the following.

Corollary 2.1 Assume that the hypotheses of Theorem 2.6 hold. If $y^{(i)}(\alpha)=0$ for $i=0,1,2$ and $y^{\prime \prime}(\beta)=0$, then

$$
\Phi_{1}(Q, r)+\Pi_{1}(p, r)+\Lambda_{1}\left(r, r^{\prime}\right)+\frac{2}{\pi} \frac{(\beta-\alpha)}{r(\beta)} A^{2}(r)\left(Q^{*}\right)^{\frac{1}{2}} \geq 1
$$

If instead $y^{(i)}(\beta)=0$ for $i=0,1,2$ and $y^{\prime \prime}(\alpha)=0$, then

$$
\Phi_{2}(Q, r)+\Pi_{2}(p, r)+\Lambda_{2}\left(r, r^{\prime}\right)+\frac{2}{\pi} \frac{(\beta-\alpha)}{r(\beta)} A^{2}(r)\left(Q^{*}\right)^{\frac{1}{2}} \geq 1 .
$$

Theorem 2.7 Suppose that $y$ is a nontrivial solution of $(1.2)$ and $Q(t)$ is the antiderivative of $q$. If

$$
y(\alpha)=y^{\prime}(\alpha)=y(\beta)=y^{\prime}(\beta)=0,
$$


then

$$
4 A^{2}(r)+\max _{t \in[\alpha, \beta]}|Q(t)| \int_{\alpha}^{\beta} \frac{(s-\alpha)^{2}}{r(s)} d s \geq 1
$$

where $A$ is defined as in (2.9).

Proof Multiplying (1.2) by $y(t)$ and integrating by parts the left-hand side twice and using (2.37), we have

$$
\int_{\alpha}^{\beta} r(t)\left|y^{\prime \prime}(t)\right|^{2} d t=\int_{\alpha}^{\beta}\left(p(t) y^{\prime}(t)\right)^{\prime} y(t) d t-\int_{\alpha}^{\beta} q(t) y^{2} d t .
$$

Using the assumption $Q^{\prime}(t)=q(t)$, we get that

$$
\int_{\alpha}^{\beta} r(t)\left|y^{\prime \prime}(t)\right|^{2} d t=\int_{\alpha}^{\beta}\left(p(t) y^{\prime}(t)\right)^{\prime} y(t) d t-\int_{\alpha}^{\beta} Q^{\prime}(t) y^{2}(t) d t .
$$

Integrating by parts the term $\int_{\alpha}^{\beta} Q^{\prime}(t) y^{2}(t) d t$, we see that

$$
\int_{\alpha}^{\beta} Q^{\prime}(t) y^{2}(t) d t=\left.Q(t) y^{2}(t)\right|_{\alpha} ^{\beta}-2 \int_{\alpha}^{\beta} Q(t) y(t) y^{\prime}(t) d t .
$$

Using the assumption $y(\alpha)=y(\beta)=0$, we see that

$$
\int_{\alpha}^{\beta} Q^{\prime}(t) y^{2}(t) d t=-2 \int_{\alpha}^{\beta} Q(t) y(t) y^{\prime}(t) d t
$$

Integrating by parts the term $\int_{\alpha}^{\beta}\left(p(t) y^{\prime}(t)\right)^{\prime} y(t) d t$, we see that

$$
\int_{\alpha}^{\beta}\left(p(t) y^{\prime}(t)\right)^{\prime} y(t) d t=\left.p(t) y^{\prime}(t) y(t)\right|_{\alpha} ^{\beta}-\int_{\alpha}^{\beta} p(t)\left(y^{\prime}(t)\right)^{2} d t .
$$

Using the assumption $y(\alpha)=y(\beta)=0$, we see that

$$
\int_{\alpha}^{\beta}\left(p(t) y^{\prime}(t)\right)^{\prime} y(t) d t=-\int_{\alpha}^{\beta} p(t)\left(y^{\prime}(t)\right)^{2} d t
$$

Substituting (2.41) and (2.42) into (2.40), we have

$$
\int_{\alpha}^{\beta} r(t)\left|y^{\prime \prime}(t)\right|^{2} d t \leq \int_{\alpha}^{\beta}|p(t)|\left|y^{\prime}(t)\right|^{2} d t+2 \int_{\alpha}^{\beta}|Q(t)||y(t)|\left|y^{\prime}(t)\right| d t .
$$

Applying the inequality (2.25) on the integral

$$
\int_{\alpha}^{\beta}|Q(t)||y(t)|\left|y^{\prime}(t)\right| d t
$$


with $c(t)=Q(t), k=0$ and $n=2$, we see that

$$
\begin{aligned}
& \int_{\alpha}^{\beta}|Q(t)||y(t)|\left|y^{\prime}(t)\right| d t \\
& \quad \leq \frac{1}{2} \max _{t \in[\alpha, \beta]}|Q(t)| \int_{\alpha}^{\beta} \frac{(s-\alpha)^{2}}{r(s)} d s\left[\int_{\alpha}^{\beta} r(t)\left|y^{\prime \prime}(t)\right|^{2} d t\right],
\end{aligned}
$$

where $y(\alpha)=y^{\prime}(\alpha)=0$. Applying the inequality (2.6), we see that

$$
\int_{\alpha}^{\beta}|p(t)|\left|y^{\prime}(t)\right|^{2} d t \leq 4 A^{2}(r) \int_{\alpha}^{\beta} r(t)\left|y^{\prime \prime}(t)\right|^{2} d t .
$$

Substituting (2.44) and (2.45) into (2.43), we have

$$
4 A^{2}(r)+\max _{t \in[\alpha, \beta]}|Q(t)| \int_{\alpha}^{\beta} \frac{(s-\alpha)^{2}}{r(s)} d s \geq 1,
$$

which is the desired inequality (2.38). The proof is complete.

Remark 2 One could consider the condition $y(\beta)=y^{\prime}(\beta)=0$ instead of $y(\alpha)=y^{\prime}(\alpha)=0$ in the proof of Theorem 2.8. In this case, the term $P_{1}(t)=\int_{\alpha}^{t} \frac{1}{r(s)} d s$ is replaced by $P_{2}(t)=$ $\int_{t}^{\beta} \frac{1}{r(s)} d s$, and also the term $\int_{\alpha}^{\beta} \frac{(s-\alpha)^{2}}{r(s)} d s$ is replaced by $\int_{\alpha}^{\beta} \frac{(\beta-s)^{2}}{r(s)} d s$.

By using $\max _{t \in[\alpha, \beta]}|p(t)|$ instead of $p(t)$ in the proof of Theorem 2.7, we have the following result.

Theorem 2.8 Suppose that $y$ is a nontrivial solution of $(1.2)$ and $Q(t)$ is the antiderivative of $q(t)$. If $y(\alpha)=y^{\prime}(\alpha)=y(\beta)=y^{\prime}(\beta)=0$, then

$$
4 \max _{t \in[\alpha, \beta]}|p(t)| \frac{(\beta-\alpha)^{2}}{3}+\max _{t \in[\alpha, \beta]}|Q(t)| \frac{(\beta-\alpha)^{3}}{6 r(\beta)} \geq 1
$$

where $A$ is defined as in (2.9).

Remark 3 The contrapositive of the results in Theorems 2.7 and 2.8 yields sufficient conditions for $(2,2)$-disconjugacy of equation (1.2).

Remark 4 One can consider the boundary conditions $y(\alpha)=y^{\prime}(\alpha)=y^{\prime \prime}(\beta)=r(\beta) y^{\prime \prime \prime}(\beta)-$ $p(\beta) y^{\prime}(\beta)=0$, which correspond to a beam hinged or supported at both ends. The proof is similar to the proof of Theorem 2.7. The details are left to the interested reader.

\section{Examples}

The following examples illustrate the results.

Example 1 Consider the equation

$$
\left(\frac{1}{t} y^{\prime \prime}(t)\right)^{\prime \prime}+A \cos (\gamma t) y(t)=0, \quad 0<\alpha \leq t \leq \beta
$$


where $A$ and $\gamma$ are positive constants. If $y(t)$ is a solution of (3.1) with $y^{(i)}(\alpha)=y^{(i)}(\beta)=0$ for $i=0,1$, then the condition (2.46) of Theorem 2.8 reads

$$
\max _{t \in[\alpha, \beta]}|Q(t)| \frac{\beta(\beta-\alpha)^{3}}{6} \geq 1,
$$

i.e.

$$
\frac{A}{\gamma}\left[\frac{\beta(\beta-\alpha)^{3}}{6}\right] \geq 1
$$

Example 2 Consider the equation

$$
y^{(4)}(t)+\lambda \cos (\gamma t) y(t)=0, \quad 0 \leq t \leq \beta,
$$

where $\lambda$ and $\alpha$ are positive constants, with $y^{(i)}(0)=0$, for $i=0,1,2$ and $y^{\prime \prime}(\beta)=0$. Then Theorem 2.2 gives us that

$$
\frac{1}{8}\left[\int_{\alpha}^{\beta} Q^{2}(t)\left[\int_{t}^{\beta}(s-t)^{4} d s\right] d t\right]=\frac{1}{40} \int_{0}^{\beta} \frac{\lambda^{2}}{\gamma^{2}} \int_{0}^{\beta} \sin ^{2}(\gamma t)(\beta-t)^{5} d t \geq 1,
$$

so

$$
\frac{\lambda^{2} \beta^{5}}{40 \gamma^{2}} \geq \frac{1}{40} \int_{0}^{\beta} \frac{\lambda^{2}}{\gamma^{2}} \int_{0}^{\beta} \sin ^{2}(\gamma t)(\beta-t)^{5} d t \geq 1,
$$

i.e.

$$
\beta>\left(\frac{40}{\lambda^{2}}\right)^{\frac{1}{5}} \gamma^{\frac{2}{5}}
$$

From this we conclude that the interval of disconjugacy is bounded below by constant times $\gamma^{\frac{2}{5}}$ for $\gamma \geq 1$, i.e., if $[0, \beta)$ is the interval of disconjugacy, then $\beta<\left(\frac{40}{\lambda^{2}}\right)^{\frac{1}{5}} \gamma^{\frac{2}{5}}$.

\section{Competing interests}

The authors declare that they have no competing interest.

Authors' contributions

Each author contributed equally to the paper. All authors read and approved the final version.

\section{Author details}

'Department of Mathematics, Faculty of Science, Mansoura University, Mansoura, 35516, Egypt. ${ }^{2}$ Department of Mathematics, Texas A\&M University-Kingsville, Kingsville, Texas 78363, USA. ${ }^{3}$ Department of Mathematics, Faculty of Science, King Abdulaziz University, Jeddah, 21589, Saudi Arabia. ${ }^{4}$ School of Mathematics, Statistics and Applied Mathematics, National University of Ireland, Galway, Ireland.

Received: 14 March 2013 Accepted: 11 May 2013 Published: 3 June 2013

\section{References}

1. Courant, R, Hilbert, D: Methods of Mathematical Physics, vol. I. Wiley, New York (1989)

2. Zill, DG, Wright, WS, Cullen, MR: Advanced Engineering Mathematics, 4th edn. Jones \& Bartlett, Boston (2011)

3. Ahmad, S: Asymptotic properties of linear fourth order differential equations. Proc. Am. Math. Soc. 59, 45-51 (1976)

4. Amara, J, Vladimirov, AA: On oscillation of eigenfunctions of fourth order problem with spectral parameters in the boundary conditions. J. Math. Sci. 150, 2317-2325 (2008)

5. Amara, J: Oscillation for fourth-order differential equations with middle term. Math. Nachr. 285, $42-46$ (2012) 
6. Hou, C, Cheng, SS: Asymptotic dichotomy in a class of fourth-order nonlinear delay differential equations with damping. Abstr. Appl. Anal. 2009, Article ID 484158 (2009)

7. Jaroš, J: Comparison theorems for half-linear differential equations of fourth order. Acta Math. Univ. Comen. LXXX 279-284 (2011)

8. Kreith, K: Nonselfadjoint fourth order differential equations with conjugate points. Bull. Am. Math. Soc. 80, 1190-1192 (1974)

9. Peterson, A: The distribution of zeros of extremal solutions of a fourth order differential equation for the $n$-th conjugate point. J. Differ. Equ. 8, 502-511 (1970)

10. Peterson, A: Distribution of zeros of fourth order differential equations. Pac. J. Math. 30, 751-764 (1969)

11. Schneider, LJ: Oscillation properties of the $2-2$ disconjugate fourth order selfadjoint differential equation. Proc. Am. Math. Soc. 28, 545-550 (1971)

12. Sobalová, M: On oscillatory solutions of the fourth order differential equations with the middle term. Nonlinear Anal. 47, 3573-3578 (2001)

13. Taylor, WE Jr.: Asymptotic behavior of solutions of a fourth order nonlinear differential equation. Proc. Am. Math. Soc. 65, 70-72 (1977)

14. Taylor, WE Jr.: Qualitative properties of solutions of certain fourth order linear differential equations. Int. J. Math. Math Sci. 4, 763-774 (1981)

15. Taylor, WE Jr.: On the oscillatory and asymptotic behavior of solutions of a certain fourth order linear differential equation. Hiroshima Math. J. 7, 667-674 (1977)

16. Taylor, WE Jr.: Oscillation criteria for certain nonlinear fourth order equations. Int. J. Math. Math. Sci. 6(3), 551-557 (1983)

17. Zhang, M, Sun, J, Ao, J: Oscillation criteria of a class of fourth order differential equations. Math. Methods Appl. Sci. (2012). doi:10.1002/mma.1583

18. Brown, R, Hinton, D: Lyapunov inequalities and their applications. In: Rassias, T (ed.) Survey on Classical Inequalities, pp. 1-25. Kluwer Academic, Dordrecht (2000)

19. Clark, S, Hinton, D: Some disconjugacy criteria for differential equations with oscillatory coefficients. Math. Nachr. 278 1476-1489 (2005)

20. Saker, SH: Lyapunov's type inequalities for fourth order differential equations. Abstr. Appl. Anal. 2012, Article ID 795825 (2012). doi:10.1155/2012/795825

21. de la Valle Poussin, C: Sur I 'equation differentielle du second ordre. J. Math. Pures Appl. 9, 125-144 (1929)

22. Agarwal, RP, Pang, PYH: Opial Inequalities with Applications in Differential and Difference Equations. Kluwer Academic, Dordrecht (1995)

23. Kufner, A, Persson, L-E: Weighted Inequalities of Hardy Type. World Scientific, River Edge (2003)

24. Kufner, A, Maligranda, L, Persson, L-E: The Hardy Inequalities: About Its History and Some Related Results. Vydavatelský Servis, Pilsen (2007)

25. Agarwal, RP, Pang, PYH: Sharp Opial-type inequalities involving higher order derivatives of two functions. Math. Nachr. 174, 5-20 (1995)

26. Boyd, D: Best constants in class of integral inequalities. Pac. J. Math. 30, 367-383 (1969)

doi:10.1186/1029-242X-2013-278

Cite this article as: Saker et al.: Properties of solutions of fourth-order differential equations with boundary conditions. Journal of Inequalities and Applications 2013 2013:278.

\section{Submit your manuscript to a SpringerOpen ${ }^{\circ}$ journal and benefit from:}

- Convenient online submission

Rigorous peer review

- Immediate publication on acceptance

- Open access: articles freely available online

- High visibility within the field

- Retaining the copyright to your article 